\title{
Quantification of aerosol dispersal from suspected aerosol-generating procedures
}

\author{
Runar Strand-Amundsen $\mathbb{1}^{1}$, Christian Tronstad ${ }^{1}$, Ole Elvebakk ${ }^{1}$, Tormod Martinsen ${ }^{1}$, Marius Dybwad ${ }^{2}$, \\ Egil Lingaas ${ }^{3}$ and Tor Inge Tønnessen ${ }^{4,5}$
}

${ }^{1}$ Dept of Clinical and Biomedical Engineering, Oslo University Hospital, Oslo, Norway. ${ }^{2}$ Norwegian Defence Research Establishment (FFI), Kjeller, Norway. ${ }^{3}$ Dept of Infection Prevention, Oslo University Hospital - Rikshospitalet, Oslo, Norway. ${ }^{4}$ Dept of Anaesthesiology, Division of Emergencies and Critical Care, Oslo University Hospital, Oslo, Norway. ${ }^{5}$ Institute of Clinical Medicine, Faculty of Medicine, University of Oslo, Oslo, Norway.

Corresponding author: Runar Strand-Amundsen (runar.strand-amundsen@fys.uio.no)

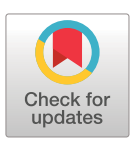

Copyright @The authors 2021

This version is distributed under the terms of the Creative Commons Attribution NonCommercial Licence 4.0. For commercial reproduction rights and permissions contact permissions@ersnet.org

This article has supplementary material available from openres.ersjournals.com

Received: 22 March 2021 Accepted: 11 Sept 2021

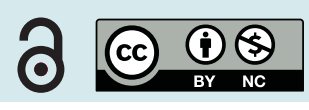

Shareable abstract (@ERSpublications)

High-flow nasal cannula or NIV oxygen treatment does not lead to an increase in aerosol dispersal compared to the use of low-flow nasal cannula oxygen. For a coughing patient, using dual-limb NIV may reduce the risk of aerosol spread. https://bit.ly/3AnoyJu

Cite this article as: Strand-Amundsen R, Tronstad C, Elvebakk O, et al. Quantification of aerosol dispersal from suspected aerosol-generating procedures. ERJ Open Res 2021; 7: 00206-2021 [DOI: 10.1183/23120541.00206-2021].

\section{Abstract}

Background Oxygen-delivering modalities like humidified high-flow nasal cannula (HFNC) and noninvasive positive-pressure ventilation (NIV) are suspected of generating aerosols that may contribute to transmission of disease such as coronavirus disease 2019. We sought to assess if these modalities lead to increased aerosol dispersal compared to the use of non-humidified low-flow nasal cannula oxygen treatment (LFNC).

Methods Aerosol dispersal from 20 healthy volunteers using HFNC, LFNC and NIV oxygen treatment was measured in a controlled chamber. We investigated effects related to coughing and using a surgical face mask in combination with the oxygen delivering modalities. An aerodynamic particle sizer measured aerosol particles (APS3321, 0.3-20 $\mu \mathrm{m}$ ) directly in front of the subjects, while a mesh of smaller particle sensors (SPS30, 0.3-10 $\mu \mathrm{m}$ ) was distributed in the test chamber.

Results Non-productive coughing led to significant increases in particle dispersal close to the face when using LFNC and HFNC but not when using NIV. HFNC or NIV did not lead to a statistically significant increase in aerosol dispersal compared to LFNC. With non-productive cough in a room without air changes, there was a significant drop in particle levels between $100 \mathrm{~cm}$ and $180 \mathrm{~cm}$ from the subjects.

Conclusions Our results indicate that using HFNC and NIV does not lead to increased aerosol dispersal compared to low-flow oxygen treatment, except in rare cases. For a subject with non-productive cough, NIV with double-limb circuit and non-vented mask may be a favourable choice to reduce the risk for aerosol spread.

\section{Introduction}

For coronavirus disease 2019 (COVID-19) patients experiencing reduced lung function with insufficient oxygenation, using humidified high-flow nasal cannula oxygenation (HFNC) and noninvasive ventilation (NIV) have been reported to reduce the frequency of intubation and subsequent invasive mechanical ventilation [1]. HFNC has been associated with a reduction in intensive care unit length of stay [2]. As these treatment modalities are suspected to be aerosol-generating procedures [3], some medical centres were initially reluctant to use them and thereby lowered the threshold for intubation. With increasing numbers of COVID-19 patients overwhelming the capacity of intensive care unit beds with invasive ventilation, many patients have been treated in stepdown units with HFNC or NIV. For the health and safety of healthcare workers, it is important to assess to what extent these procedures generate aerosols. Documentation for airborne transmission being a component for the spread of COVID-19 is surfacing [4, 5]. At the same time, there are reports that the potential viral load of aerosolised particles may be low [6]. 
Several recent approaches have investigated aerosol dispersal and spread during oxygen therapies, and research is still ongoing to answer these questions [7-13]. While the present evidence is non-conclusive on whether HFNC or NIV oxygen treatment has high aerosol dispersal potential, results from recent studies suggest that they do not lead to significantly increased aerosol dispersal compared to low-flow oxygen modalities [14-17].

In our study aerosol levels were measured in a controlled chamber, where 20 healthy volunteers used HFNC, non-humidified low-flow nasal cannula (LFNC) and NIV in biphasic positive airway pressure (BIPAP) mode. We investigated the parameter "coughing” with all oxygen modalities, and for HFNC we also investigated using a "surgical face mask". An aerodynamic particle sizer was used to measure (optical) aerosol particles $(0.3-20 \mu \mathrm{m})$ directly in front $(30 \mathrm{~cm})$ of the subject, while smaller optical particle sensors $(0.3-10 \mu \mathrm{m})$ were distributed in the test chamber.

We sought to assess the following questions:

1. Does the use of HFNC or NIV lead to increased aerosol dispersal compared to the use of LFNC?

2. How does non-productive coughing contribute to aerosol dispersal in settings with HFNC/NIV/LFNC?

3. How is the spatial distribution of aerosols in a confined space influenced by HFNC/NIV/LFNC?

\section{Methods}

We recruited healthy adult volunteers aged $>18$ years. The recruitment process and study were approved by the regional committee for medical and health research ethics in Norway (approval reference: REK 153325), and written consent was obtained from the subjects.

\section{Experimental protocol}

The protocol comprised three oxygen modalities, LFNC, HFNC and NIV (figure 1b), split into eight events. We included coughing events with all modalities and included a test with a surgical face mask (Medline, EN14683 Type II, Cheshire, England) within the HFNC modality. The subjects were instructed to provoke coughing (voluntary intensity) every $30 \mathrm{~s}$ during the coughing tests. To counter potential carryover effects by protocol sequence, the modality order was separated into four differently ordered subsets where the 20 subjects were divided evenly. For LFNC and HFNC we used an Airvo 2 (Fisher \& Paykel Healthcare Limited, Auckland, New Zealand) with an Optiflow nasal cannula (Fisher \& Paykel Healthcare Limited, Auckland, New Zealand). The following flowrates were used: $\mathrm{LFNC}=4 \mathrm{~L} \cdot \mathrm{min}^{-1}$, HFNC=increments of $10 \mathrm{~L} \cdot \mathrm{min}^{-1}$, starting at 10 and ending at $60 \mathrm{~L} \cdot \mathrm{min}^{-1}$. For NIV (dual-limb) in BIPAP mode (spontaneous, IPAP 10, EPAP 5, support frequency 4) we used a Hamilton C6 (Hamilton Medical, Bonaduz, Switzerland) with silicone facial masks (Respireo Hospital F, NonVented disposable, Air Liquide Medical Systems, Antony, France).

\section{Test chamber}

The test chamber (figure 1a) with no external active air supply or internal air currents had an internal volume of $11.36 \mathrm{~m}^{3}$ (l:w:h: $234.5 \times 234.5 \times 206.5 \mathrm{~cm}$ ), with an anteroom to reduce aerosol contamination. A City M Air Purifier (CAMFIL, Riverdale, NJ, USA) with an airflow of $7.2 \mathrm{~m}^{3} \cdot \mathrm{min}^{-1}$ was used to zero the particle levels before and between each event. Each subject wore a disposable polypropylene non-woven coverall (Worksafe).

\section{Measurement and equipment}

Two different classes of aerosol instruments were used to count and measure particle sizes. A TSI model 3321 Aerodynamic Particle Sizer ${ }^{\circledR}$ (TSI Incorporated, Shoreview, MN, USA) was used to sample the air in the breathing-zone $30 \mathrm{~cm}$ in front of the subject. Nine small particle sensors (SPS30, Sensirion AG, Staefa ZH, Switzerland) were used (custom-made wireless setup) for simultaneous particle counting at different locations (figure 1a). Both instruments measure the aerosol concentration over a range of particle sizes, from $0.3 \mu \mathrm{m}$ (optical size) or $0.5 \mu \mathrm{m}$ (aerodynamic size) to $20 \mu \mathrm{m}$ for the APS 3321, and from $0.3 \mu \mathrm{m}$ (optical size) to $10 \mu \mathrm{m}$ for the SPS30. For readability we use the name "breathing-zone single sensor" for the APS 3321, and "mesh sensors" for the SPS30 sensors.

Prior to performing the experiments, the particle sensors were tested with particle generation from humans and with a $\mathrm{NaCl}$ aerosol generator. Temperature and relative humidity in the test chamber were continuously logged during the experiments with an AM2320 sensor (Guangzhou Aosong Electronics Co., Ltd, Guangzhou, China). There was low variation with a mean of 26.0 \pm 0.7 STD ${ }^{\circ} \mathrm{C}$ and 47.1 \pm 5.6 STD \% relative humidity. 


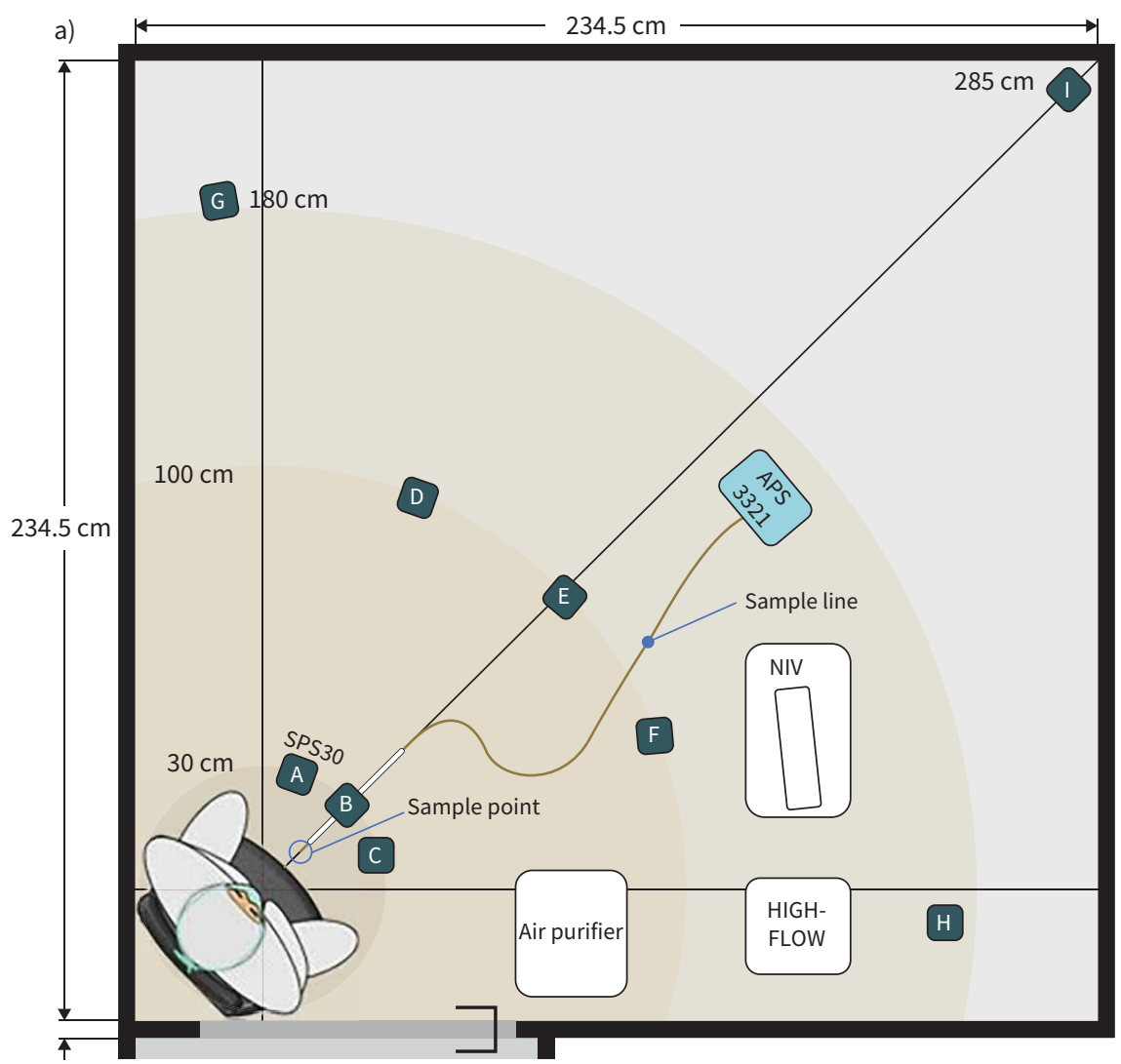

b)

\begin{tabular}{|c|c|c|c|}
\hline \# Modalit & Elements & Details & Duration (min) \\
\hline 1 LFNC & & LFNC - airflow: $4 \mathrm{~L} \cdot \mathrm{min}^{-1}$ & 10 \\
\hline 2 LFNC & Cough & LFNC - airflow: $4 \mathrm{~L} \cdot \mathrm{min}^{-1}$ (cough every 30 seconds) & 10 \\
\hline 3 HFNC & & $\mathrm{HF}$ increased stepwise $10-60 \mathrm{~L} \cdot \mathrm{min}^{-1}$ over the first $3 \mathrm{~min}$ & 10 \\
\hline HFNC & Cough & $\mathrm{HF}$ increased stepwise $10-60 \mathrm{~L} \cdot \mathrm{min}^{-1}$ over the first $3 \mathrm{~min}$ (cough every 30 seconds) & 10 \\
\hline 5 HFNC & Mask & $\mathrm{HF}$ increased stepwise $10-60 \mathrm{~L} \cdot \mathrm{min}^{-1}$ over the first $3 \mathrm{~min}$ & 10 \\
\hline 6 HFNC & Cough, mask & $\mathrm{HF}$ increased stepwise $10-60 \mathrm{~L} \cdot \mathrm{min}^{-1}$ over the first $3 \mathrm{~min}$ (cough every 30 seconds) & 10 \\
\hline NIV & & Spontaneous, IPAP 10, EPAP 5, support freq $=4$ & 10 \\
\hline 8 NIV & Cough & Spontaneous, IPAP 10, EPAP 5 , support freq $=4$ (cough every 30 seconds) & 10 \\
\hline
\end{tabular}

FIGURE 1 a) Overview of the test chamber, sensor locations (Sensirion, SPS30: mesh sensors: A-I, three sensors at $30 \mathrm{~cm}$, three at $100 \mathrm{~cm}$, two at $180 \mathrm{~cm}$ and one at $285 \mathrm{~cm}$ ) (TSI, APS 3321: "breathing zone single sensor": sample point $20 \mathrm{~cm}$ from participant face) and other equipment. The test person was seated on a chair, with an approximate breathing zone elevation of $120 \mathrm{~cm}$ above the floor. The sensors were positioned $120 \mathrm{~cm}$ above the floor. b) Protocol structure with oxygen modalities and event elements and details. Before and between each 10-min event a 15-min period of filtering out the particles of the test chamber was performed. LFNC: low-flow nasal cannula; HFNC: high-flow nasal cannula; NIV: noninvasive positive-pressure ventilation. HF: high flow; IPAP: inspiratory positive airway pressure; EPAP: expiratory positive airway pressure

\section{Data analysis and statistics}

Comparisons were made of the particle concentration between all pairwise combinations of events relevant to the research questions. As the distributions in concentration values were highly skewed and deviated from a normal distribution (confirmed by Shapiro-Wilk test), comparisons of medians were conducted. To quantify differences between events, median differences were calculated, and their 95\% confidence intervals were estimated using the bootstrap method. The Wilcoxon signed rank test was used to quantify the statistical significance of the differences. These comparisons were done on both datasets (breathing-zone single sensor and mesh sensors).

To obtain statistical estimates of changes in particle concentration over time, the particle concentration was modelled as a function of time using a linear mixed-effects model with random intercept and slope, using 
the averaged particle concentrations over each minute as model input. A full covariance matrix based on Cholesky parameterisation was used as covariance structure in the models, selected based on the Akaike information criterion on models fit on the breathing-zone single sensor dataset. The particle concentration dependency on the four distance-categories of the mesh sensors (figure 1a) was modelled statistically for each event using a linear mixed-effects model with random intercept and fixed slope. Estimation of Spearman correlations between emitted particle levels and age, sex and weight were done in Graphpad 9.0.0. All other statistical calculations were done in Matlab R2019b.

\section{Results}

\section{Descriptive statistics}

20 healthy adult subjects were included in the study: seven were female, mean \pm sD age was $43 \pm 11.3$ years and mean \pm SD weight was $80 \pm 18.7 \mathrm{~kg}$. Descriptive statistics for the measurements by the breathing-zone single sensor are presented in table 1 . As visualised in figure $2 a$ and $b$, there was a large spread in the particle concentration among subjects, with a consistent dispersion around $100 \%$ for most events. The distributions were positively skewed with tails of extreme values. Most of the particles measured by the breathing-zone single sensor were in the range of $0.3-5 \mu \mathrm{m}$ (figure 2e). Our measurement-setup fulfilled the criteria for representative aerosol sampling (intake) and high transport efficiency (tubing) for particle sizes $\leqslant 5 \mu \mathrm{m}$. The particle dispersal from coughing produced spiked measurements, with the largest spikes attributed to only a few individuals (figure 3 and supplementary Figures E1-E3). No correlations were found between aerosol dispersion and age, sex or weight.

\begin{tabular}{|c|c|c|c|c|c|c|c|}
\hline Event & Mean & Min & Max & Median & 0.25 & 0.75 & Dispersion \% \\
\hline \multicolumn{8}{|c|}{ Particle size $\leqslant 1.0 \mu \mathrm{m}$} \\
\hline LFNC & 73.0 & 21.2 & 151.0 & 60.9 & 41.5 & 106.5 & 107 \\
\hline LFNC+cough & 222.7 & 44.9 & 1167.4 & 122.9 & 71.0 & 248.4 & 144 \\
\hline HFNC & 101.2 & 15.9 & 317.1 & 67.8 & 38.5 & 130.0 & 135 \\
\hline HFNC+cough & 190.9 & 36.4 & 1444.7 & 91.9 & 61.8 & 167.6 & 115 \\
\hline $\mathrm{HFNC}+\mathrm{M}$ & 89.3 & 25.3 & 230.5 & 54.8 & 41.0 & 137.2 & 176 \\
\hline $\mathrm{HFNC}+\mathrm{M}+$ cough & 103.1 & 29.2 & 270.5 & 96.7 & 48.2 & 156.5 & 112 \\
\hline NIV & 77.7 & 20.6 & 245.9 & 66.4 & 33.3 & 102.4 & 104 \\
\hline NIV+cough & 90.2 & 28.0 & 263.5 & 73.8 & 39.2 & 112.2 & 99 \\
\hline \multicolumn{8}{|c|}{ Particle size $>1 \mu \mathrm{m}$ and $\leqslant 5 \mu \mathrm{m}$} \\
\hline LFNC & 22.1 & 7.3 & 70.2 & 18.6 & 14.8 & 24.0 & 49 \\
\hline LFNC+cough & 47.3 & 13.7 & 242.0 & 29.3 & 17.7 & 40.4 & 77 \\
\hline HFNC & 42.4 & 1.8 & 236.7 & 25.7 & 14.9 & 46.0 & 121 \\
\hline HFNC+cough & 41.8 & 13.3 & 186.4 & 25.0 & 21.4 & 37.7 & 65 \\
\hline $\mathrm{HFNC}+\mathrm{M}$ & 33.5 & 7.6 & 110.2 & 22.4 & 15.4 & 39.4 & 107 \\
\hline $\mathrm{HFNC}+\mathrm{M}+$ cough & 27.3 & 8.4 & 80.1 & 23.4 & 17.3 & 29.1 & 50 \\
\hline NIV & 21.6 & 5.1 & 51.8 & 22.1 & 13.3 & 26.4 & 59 \\
\hline NIV+cough & 24.7 & 6.0 & 72.7 & 21.6 & 16.3 & 31.2 & 69 \\
\hline \multicolumn{8}{|l|}{ Particle size $>5 \mu \mathrm{m}$} \\
\hline LFNC & 1.7 & 0.5 & 5.5 & 1.2 & 0.9 & 2.1 & 100 \\
\hline LFNC+cough & 1.7 & 0.3 & 5.5 & 1.4 & 1.0 & 1.8 & 57 \\
\hline HFNC & 3.6 & 0.1 & 22.8 & 1.9 & 1.2 & 3.5 & 122 \\
\hline HFNC+cough & 3.1 & 0.6 & 24.6 & 1.6 & 1.1 & 2.9 & 113 \\
\hline $\mathrm{HFNC}+\mathrm{M}$ & 3.7 & 0.4 & 20.5 & 1.7 & 1.1 & 3.9 & 167 \\
\hline $\mathrm{HFNC}+\mathrm{M}+$ cough & 2.6 & 0.8 & 9.8 & 1.9 & 1.2 & 2.9 & 88 \\
\hline NIV & 1.3 & 0.3 & 2.5 & 1.3 & 0.9 & 1.8 & 65 \\
\hline NIV+cough & 1.8 & 0.5 & 4.5 & 1.5 & 1.0 & 2.2 & 79 \\
\hline
\end{tabular}

Descriptive statistics for the particle concentration (three particle size groups) measured by the breathing-zone single sensor (APS 3321) during all events. The unit used is the mean particle concentration in number per litre during the event. The dispersion of the distribution is presented as the per cent-wise ratio between the interquartile range and the median. The statistical distributions of particle counts were skewed for nearly all events and particle sizes (Shapiro-Wilk test p<0.05). LFNC: low-flow nasal cannula; HFNC: high-flow nasal cannula; NIV: noninvasive positive-pressure ventilation; M: surgical mask. 

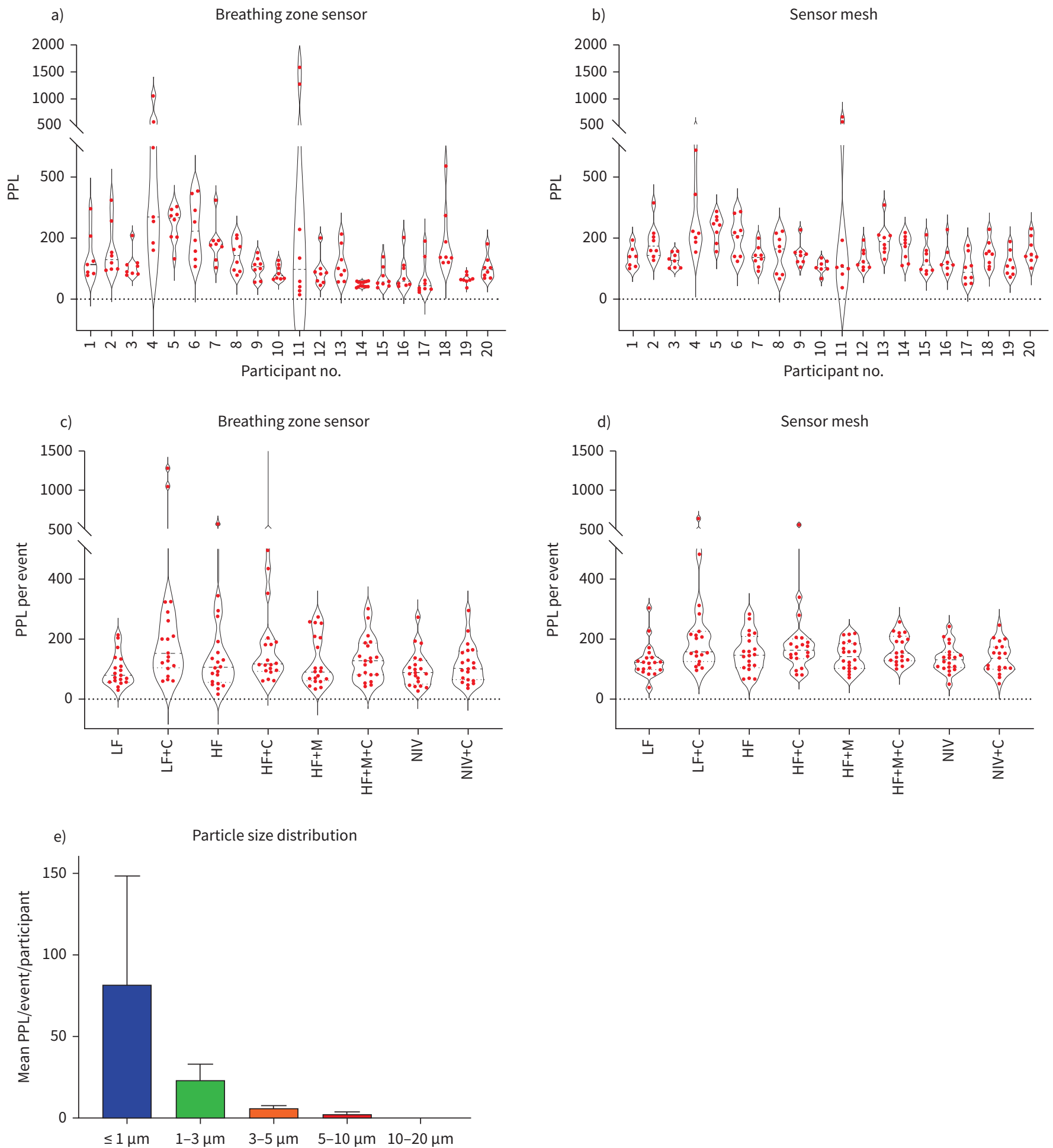

FIGURE 2 Average particles per litre of air (PPL) concentration over $10 \mathrm{~min}$ (red dot) between each of the eight events in each experiment, grouped according to participant (violin plots with two segment $y$-axes). a) Breathing-zone single sensor (particle size range $0.3-20 \mu \mathrm{m}$ ). b) average over nine mesh sensors (particle size range $0.3-10 \mu \mathrm{m}$ ). Categorical comparisons of average concentration of particles per litres of air, during the eight events of each experiment, with each red dot representing the results from 1 of 20 participants, measured by the breathing-zone single sensor (APS 3321) (c) and the average over all nine mesh sensors (d). The median is indicated with a thin black dashed line. LF: low-flow; C: cough; HF: high-flow; M: mask. e) Particle size distribution measured with the breathing-zone sensor; NIV: noninvasive positive-pressure ventilation. 
LFNC

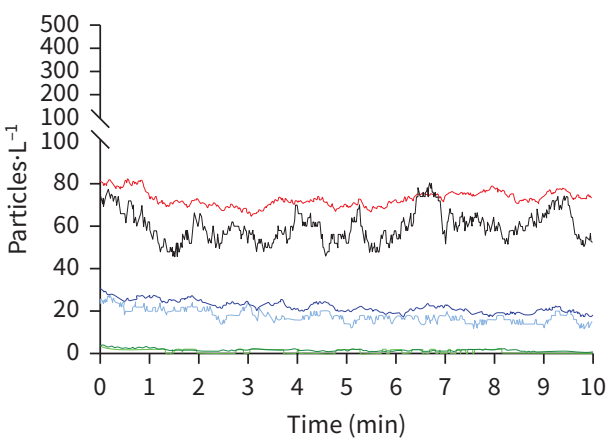

HFNC

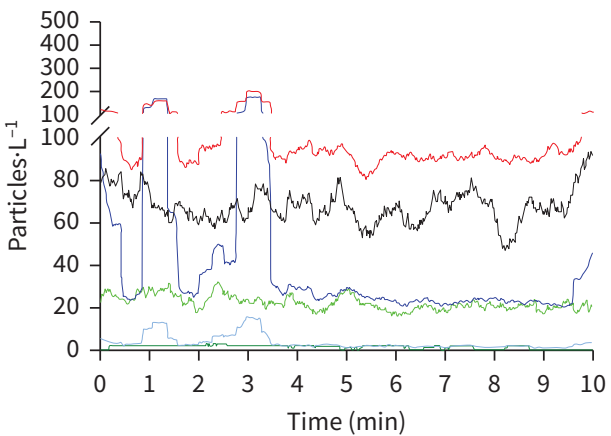

$\mathrm{HFNC}+$ mask

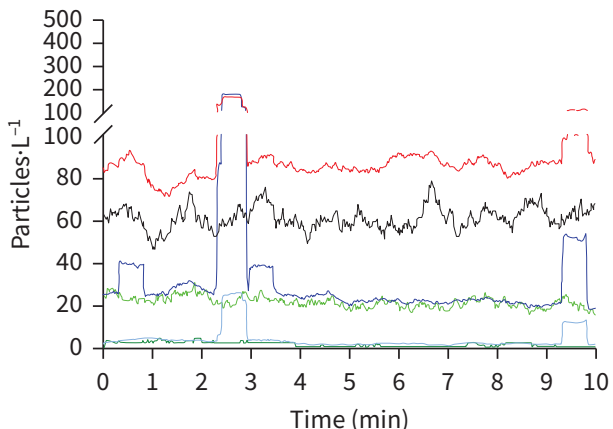

NIV

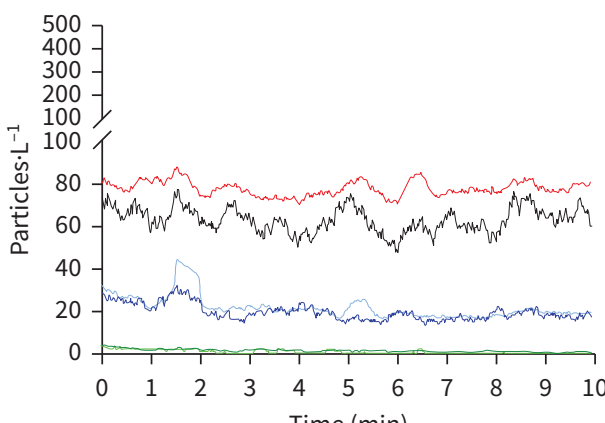
Time (min)

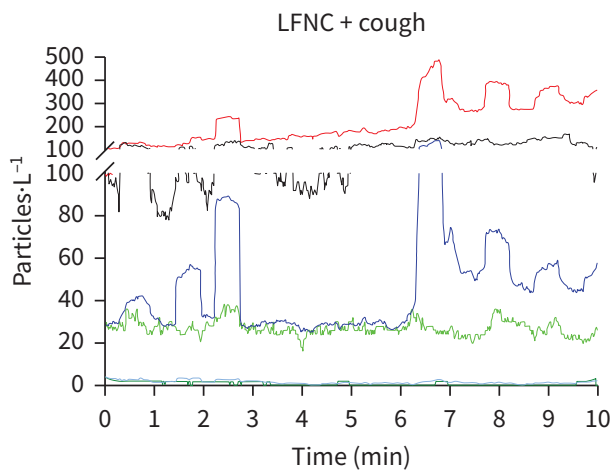

HFNC + cough

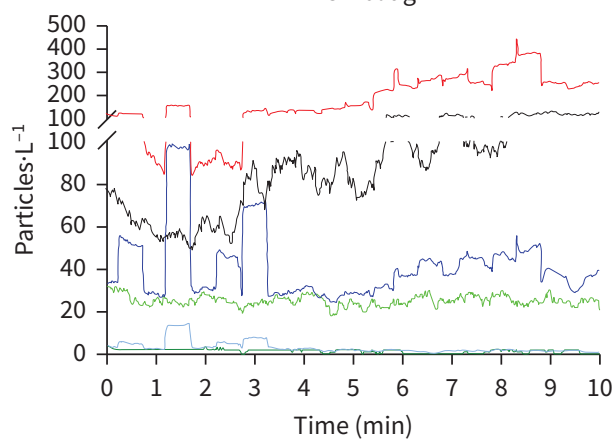

HFNC + mask + cough

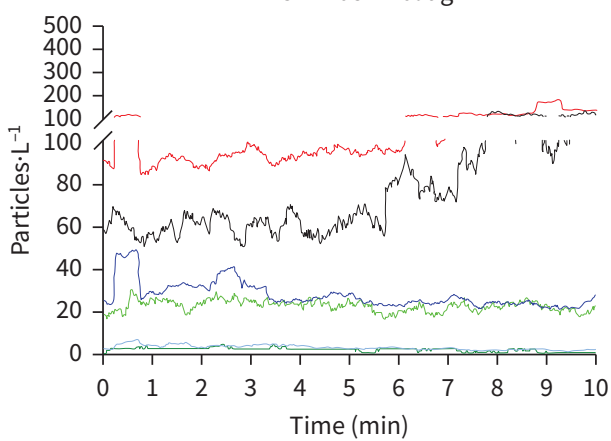

NIV + cough

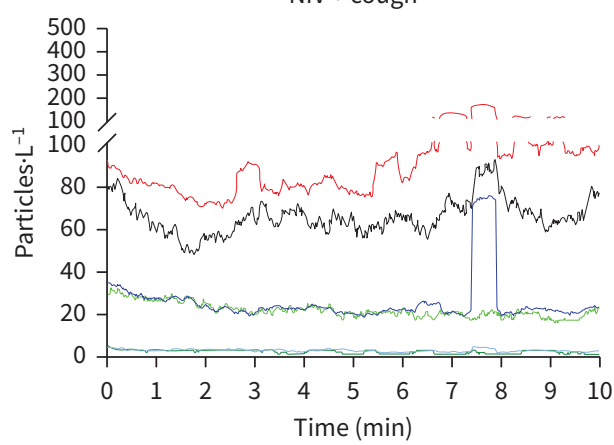

$$
\begin{aligned}
& -\leq 1 \mu \mathrm{m} \text { (mean) } \\
& \longrightarrow>1 \mu \mathrm{m} \text { and } \leq 5 \mu \mathrm{m} \text { (median) } \\
& \longrightarrow>5 \mu \mathrm{m} \text { (median) } \\
& \leq 1 \mu \mathrm{m} \text { (median) } \\
& >1 \mu \mathrm{m} \text { and } \leq 5 \mu \mathrm{m} \text { (mean) } \\
& \longrightarrow>5 \mu \mathrm{m} \text { (mean) }
\end{aligned}
$$

FIGURE 3 Mean and median particles per litre of air, from 20 participants, for three particle size groups, measured with the breathing-zone single sensor (APS 3321), during the eight events of the experiment. The median and means show smoothed time series (moving mean with a 3-s window). The smoothing was used to increase readability and instead of a series of spikes, a smoothed square-like step response is shown for the periods with large spikes. LFNC: low-flow nasal cannula; HFNC: high-flow nasal cannula; NIV: noninvasive positive-pressure ventilation. 


\begin{tabular}{|c|c|c|c|c|}
\hline Comparison & Median difference & Lower $\mathrm{Cl}$ & Upper Cl & p-value \\
\hline \multicolumn{5}{|l|}{ Particle size $\leqslant 1.0 \mu \mathrm{m}$} \\
\hline HFNC-LFNC & 9.5 & -5.3 & 29.6 & 0.212 \\
\hline LFNC+cough-LFNC & 42.9 & 8.9 & 108.5 & 0.005 \\
\hline HFNC+cough-HFNC & 8.2 & -3.4 & 31.3 & 0.145 \\
\hline HFNC+cough - LFNC+cough & -12.1 & -59.4 & -0.2 & 0.184 \\
\hline HFNC-HFNC+M & 6.7 & -8.5 & 22.1 & 0.179 \\
\hline $\mathrm{HFNC}+$ cough $-\mathrm{HFNC}+\mathrm{M}+$ cough & 11.1 & -10.9 & 35.4 & 0.126 \\
\hline $\mathrm{HFNC}+\mathrm{M}+$ cough $-\mathrm{HFNC}+\mathrm{M}$ & 2.8 & -7.0 & 14.6 & 0.332 \\
\hline NIV-LFNC & -0.7 & -4.8 & 14.2 & 0.904 \\
\hline NIV-HFNC & -4.3 & -30.3 & 7.4 & 0.204 \\
\hline NIV+cough-NIV & 6.6 & -14.5 & 16.0 & 0.455 \\
\hline LFNC+cough-NIV+cough & 43.4 & 6.0 & 94.5 & 0.001 \\
\hline HFNC+cough-NIV+cough & 28.2 & 6.4 & 43.2 & 0.023 \\
\hline \multicolumn{5}{|l|}{ Particle size $>1 \mu \mathrm{m}$ and $\leqslant 5 \mu \mathrm{m}$} \\
\hline HFNC-LFNC & 6.8 & -0.3 & 16.6 & 0.073 \\
\hline LFNC+cough-LFNC & 7.6 & -0.5 & 16.6 & 0.048 \\
\hline HFNC+cough-HFNC & 1.0 & -10.9 & 5.7 & 0.765 \\
\hline HFNC+cough - LFNC+cough & -1.4 & -7.1 & 0.0 & 0.296 \\
\hline $\mathrm{HFNC}-\mathrm{HFNC}+\mathrm{M}$ & 2.0 & -4.6 & 13.4 & 0.391 \\
\hline $\mathrm{HFNC}+$ cough $-\mathrm{HFNC}+\mathrm{M}+$ cough & 2.6 & -0.4 & 6.8 & 0.086 \\
\hline $\mathrm{HFNC}+\mathrm{M}+$ cough $-\mathrm{HFNC}+\mathrm{M}$ & 1.6 & -6.6 & 4.1 & 0.911 \\
\hline NIV-LFNC & 2.5 & -4.4 & 4.4 & 0.732 \\
\hline NIV-HFNC & -5.5 & -20.6 & -0.2 & 0.057 \\
\hline NIV+cough-NIV & 2.6 & 0.0 & 6.1 & 0.156 \\
\hline LFNC+cough-NIV+cough & 7.1 & -2.0 & 16.0 & 0.040 \\
\hline HFNC+cough-NIV+cough & 4.3 & -0.4 & 11.9 & 0.107 \\
\hline \multicolumn{5}{|l|}{ Particle size $>5 \mu \mathrm{m}$} \\
\hline HFNC-LFNC & 0.5 & 0.3 & 0.9 & 0.044 \\
\hline LFNC+cough-LFNC & 0.2 & -0.4 & 0.4 & 0.862 \\
\hline HFNC+cough-HFNC & -0.5 & -0.6 & 0.1 & 0.204 \\
\hline $\mathrm{HFNC}+$ cough $-\mathrm{LFNC}+$ cough & 0.3 & -0.1 & 0.9 & 0.116 \\
\hline $\mathrm{HFNC}-\mathrm{HFNC}+\mathrm{M}$ & 0.2 & -0.6 & 0.7 & 0.926 \\
\hline $\mathrm{HFNC}+$ cough $-\mathrm{HFNC}+\mathrm{M}+$ cough & -0.4 & -0.9 & 0.2 & 0.125 \\
\hline $\mathrm{HFNC}+\mathrm{M}+$ cough $-\mathrm{HFNC}+\mathrm{M}$ & 0.1 & -0.5 & 0.8 & 0.709 \\
\hline NIV-LFNC & -0.3 & -0.5 & 0.3 & 0.468 \\
\hline NIV-HFNC & -0.8 & -1.8 & -0.2 & 0.009 \\
\hline NIV+cough-NIV & 0.4 & 0.1 & 0.8 & 0.079 \\
\hline LFNC+cough-NIV+cough & -0.2 & -0.4 & 0.3 & 0.456 \\
\hline HFNC+cough-NIV+cough & 0.1 & -0.3 & 0.9 & 0.390 \\
\hline \multicolumn{5}{|c|}{$\begin{array}{l}\text { Statistical pairwise comparisons between different experimental events of particle concentrations (particles per } \\
\text { litre of air) measured by the breathing-zone single sensor (APS 3321). Results from three particle size groups } \\
\text { are shown. 95\% confidence intervals of the median differences are based on the bootstrap method, and the } \\
\text { p-values are based on the Wilcoxon signed rank test. LFNC: low-flow nasal cannula; HFNC: high-flow nasal } \\
\text { cannula; NIV: noninvasive positive-pressure ventilation; M: surgical mask. }\end{array}$} \\
\hline
\end{tabular}

\section{Inferential statistics}

The results from pairwise comparisons of events are shown in table 2 (breathing-zone single sensor dataset). There was a median increase in particle concentration during HFNC compared to LFNC, but the confidence intervals were wide, including changes in both directions. For particles $>5 \mu \mathrm{m}$, we measured a statistically significant difference between HFNC and LFNC, but the total particle count in this size range was small (median difference 0.5 particles/litre). Comparing events with and without coughing, there was a relatively large increase in particle concentration when coughing during LFNC, and to a lower extent during HFNC. The median particle concentration was lower with HFNC+cough compared to LFNC +cough, but this difference was not statistically significant. Compared to LFNC+cough and HFNC+cough, $\mathrm{NIV}+$ cough led to lower levels of particle dispersion, especially in the particle range $\leqslant 1.0 \mu \mathrm{m}$.

Wearing a surgical face mask reduced the mean particle concentration during HFNC, both with and without coughing, but the differences were not statistically significant. Comparing NIV with LFNC, the 
particle concentrations were similar. None of the mesh sensors measured a statistically significant difference in particle concentration between HFNC and LFNC. In agreement with the breathing-zone single sensor dataset, the largest differences were attributed to coughing, with the largest effects registered by the sensors near the breathing-zone. For details see supplementary Table E1.

\section{Concentration versus time}

Trends over time were close to zero for the events without coughing (table 3, figure 3). Although not statistically significant, the estimated trends were largest for LFNC and HFNC with coughing. With HFNC there were typically some early spikes before a slow reduction over time, resulting in slightly negative estimates. For details see supplementary Figures E1-E3. The results were similar for the three mesh sensors that were close to the subject (figure 1, supplementary Table E2).

\section{Concentration versus distance}

With the mesh sensors there were few distance-dependent differences detected except for events involving coughing (table 4, figure 4). With coughing, the differences between 30 and $100 \mathrm{~cm}$ were small, but the particle concentration was significantly reduced at 180 and $285 \mathrm{~cm}$. Notably for HFNC-M, the particle concentration was lowest at the closest distance but significantly increased $100 \mathrm{~cm}$ in front of the subject.

\section{Discussion}

We investigated the aerosol dispersal from the respiratory tract of 20 healthy adult volunteers in a test chamber with a close to zero particle baseline level, while using LFNC, HFNC or NIV, combined with the parameters "coughing" and "surgical face mask". The key findings were that HFNC or NIV did not lead to large increases in aerosol dispersal compared to LFNC, except in rare cases (3/20). When investigating changes in particle concentration at different distances, there were no large differences between LFNC, HFNC and NIV.

\begin{tabular}{|c|c|c|c|c|c|}
\hline Event & Fixed estimate & Lower $95 \% \mathrm{Cl}$ & Upper $95 \% \mathrm{Cl}$ & Intercept & p-value \\
\hline \multicolumn{6}{|c|}{ Particle size $\leqslant 1.0 \mu \mathrm{m}$} \\
\hline LFNC & 0.1 & -0.5 & 0.7 & 72.4 & 0.721 \\
\hline LFNC+cough & 27.3 & -4.0 & 58.6 & 72.5 & 0.087 \\
\hline HFNC & -2.5 & -6.7 & 1.7 & 115.1 & 0.238 \\
\hline HFNC+cough & 25.7 & -16.6 & 68.1 & 49.4 & 0.232 \\
\hline $\mathrm{HFNC}+\mathrm{M}$ & -0.2 & -2.2 & 1.9 & 90.3 & 0.862 \\
\hline $\mathrm{HFNC}+\mathrm{M}+$ cough & 4.4 & -0.3 & 9.0 & 79.0 & 0.065 \\
\hline NIV & -0.1 & -0.7 & 0.5 & 78.2 & 0.743 \\
\hline NIV+cough & 3.6 & -0.1 & 7.4 & 70.2 & 0.059 \\
\hline \multicolumn{6}{|c|}{ Particle size $>1 \mu \mathrm{m}$ and $\leqslant 5 \mu \mathrm{m}$} \\
\hline LFNC & -0.8 & -1.2 & -0.5 & 26.7 & $<0.001$ \\
\hline LFNC+cough & 2.4 & -4.2 & 8.9 & 34.2 & 0.474 \\
\hline HFNC & -5.8 & -13.2 & 1.5 & 74.5 & 0.120 \\
\hline HFNC+cough & -1.2 & -8.7 & 6.3 & 48.5 & 0.750 \\
\hline $\mathrm{HFNC}+\mathrm{M}$ & -2.6 & -7.0 & 1.7 & 48.0 & 0.235 \\
\hline $\mathrm{HFNC}+\mathrm{M}+$ cough & -1.5 & -2.9 & 0.0 & 35.4 & 0.049 \\
\hline NIV & -1.2 & -1.9 & -0.4 & 28.2 & 0.002 \\
\hline NIV+cough & 0.1 & -1.9 & 2.1 & 24.2 & 0.929 \\
\hline \multicolumn{6}{|l|}{ Particle size $>5 \mu \mathrm{m}$} \\
\hline LFNC & -0.1 & -0.2 & 0.0 & 2.4 & 0.009 \\
\hline LFNC+cough & -0.2 & -0.3 & -0.1 & 2.7 & 0.005 \\
\hline HFNC & -0.6 & -1.3 & 0.1 & 6.7 & 0.095 \\
\hline HFNC+cough & -0.6 & -1.5 & 0.3 & 6.4 & 0.180 \\
\hline $\mathrm{HFNC}+\mathrm{M}$ & -0.3 & -1.1 & 0.5 & 5.4 & 0.440 \\
\hline $\mathrm{HFNC}+\mathrm{M}+$ cough & -0.3 & -0.5 & -0.2 & 4.5 & $<0.001$ \\
\hline NIV & -0.2 & -0.3 & -0.1 & 2.5 & $<0.001$ \\
\hline NIV+cough & -0.1 & -0.2 & 0.0 & 2.3 & 0.084 \\
\hline
\end{tabular}

Estimates of change in particle concentration (number/litre) per minute near the breathing-zone of the subjects for all events, based on measurements from the breathing-zone single sensor (APS 3321). Results from three particle size groups are shown. LFNC: low-flow nasal cannula; HFNC: high-flow nasal cannula; NIV: noninvasive positive-pressure ventilation; M: surgical mask. 


\begin{tabular}{|c|c|c|c|c|}
\hline Event & $0.3 \mathrm{~m}$ (intercept) & $1.0 \mathrm{~m}$ & $1.8 \mathrm{~m}$ & $2.85 \mathrm{~m}$ \\
\hline LFNC & $218.2(168.0-268.3)$ & $22.9(-23.1-68.8)$ & $-38.0(-89.4-13.4)$ & $-14.6(-79.6-50.4)$ \\
\hline LFNC+cough & $400.2(293.1-507.3)$ & $-20.3(-84.2-43.6)$ & $-102.6(-174.1--31.1)^{*}$ & $-100.9(-191.3--10.5)^{\star}$ \\
\hline HFNC & $278.1(219.2-337.0)$ & $-30.4(-94.8-34.0)$ & $-34.8(-106.8-37.2)$ & $-16.5(-107.5-74.5)$ \\
\hline HFNC+cough & $341.8(258.6-425.0)$ & $7.1(-53.6-67.8)$ & $-99.5(-167.3--31.7)^{\star}$ & $-104.0(-189.8--18.2)^{\star}$ \\
\hline $\mathrm{HFNC}+\mathrm{M}$ & $210.5(167.2-253.9)$ & $79.1(31.5-126.7)^{\star}$ & $14.0(-39.2-67.2)$ & $25.0(-42.3-92.4)$ \\
\hline $\mathrm{HFNC}+\mathrm{M}+$ cough & $303.4(260.2-346.7)$ & $-31.0(-92.2-30.2)$ & $-57.8(-126.2-10.5)$ & $-71.3(-159.4-16.8)$ \\
\hline NIV & $222.8(183.9-261.7)$ & $35.3(-19.8-90.3)$ & $-30.2(-91.7-31.3)$ & $-20.4(-98.2-57.42)$ \\
\hline NIV+cough & $228.4(181.6-275.3)$ & $33.8(-17.5-85.0)$ & $-43.0(-100.3-14.2)$ & $9.7(-62.6-82.0)$ \\
\hline
\end{tabular}

Estimates of differences in mean particle concentration (number/L) between mesh sensors at different distances from the breathing-zone of the subject based on a linear mixed-effects model. The particle concentration number includes particle sizes $(0.3-10 \mu \mathrm{m})$. The intercept level at $30 \mathrm{~cm}$ is presented together with the differences (versus $30 \mathrm{~cm}$ ) at $100 \mathrm{~cm}, 180 \mathrm{~cm}$ and $285 \mathrm{~cm}$. 95\% confidence intervals of all estimates are given in parentheses, and all differences (versus $30 \mathrm{~cm}$ ) with a p-value $<0.05$ are marked with *. LFNC: low-flow nasal cannula; HFNC: high-flow nasal cannula; NIV: noninvasive positive-pressure ventilation; M=surgical mask.

There was a tendency for small increases in median particles during HFNC compared to LFNC, for the lower particle ranges $(<5 \mu \mathrm{m})$. HeLgeson et al. [14] reported a similar non-significant increase at $4 \mathrm{~cm}$ from the mouth of the subjects when using HFNC or oxymask. For the particle range $>5 \mu \mathrm{m}$ we measured a statistically significant difference at $30 \mathrm{~cm}$, but the overall number of particles measured in this range was low, with a median difference of just 0.5 particles per litre (table 2). The main contribution to this increase came from three subjects that dispersed more particles (of all sizes measured) when using HFNC compared to LFNC (table 2, figure 2c and d), indicating that in rare cases healthcare workers could expect patients connected to HFNC to disperse more particles. Comparing NIV with LFNC, the particle concentrations were similar, indicating that NIV did not lead to increased aerosol dispersal (table 2, figure 2c and d). The mesh sensors dataset showed similar results for pairwise comparisons between events for all sensors (supplementary Table E1). There was a trend with HFNC with spikes during the first minutes (supplementary Figures E1-E3) that could be related to the nasal irritation that some of the subjects (30\%) reported during this period. This nasal irritation might explain the slightly higher median with HFNC compared to LFNC.

Our results generally agree with recent studies that indicate that HFNC does not lead to significantly increased aerosol dispersal compared to LFNC in a group of healthy adults [7-9, 11]. The large spread in individual aerosol dispersal among the 20 subjects (figure 2), despite measures to reduce confounders and background noise, indicates that there are strong individual factors influencing aerosol dispersal. To compare the extremes, the individual with the lowest aerosol dispersal produced a mean of 52 particles $\cdot \mathrm{L}^{-1}$ for the eight events of the protocol, while the individual with the highest aerosol dispersal produced a mean of 425 particles $\cdot \mathrm{L}^{-1}$.

We used a controlled chamber and zeroed particles between each event to ensure accurate measurement of aerosol dispersal from the subjects without dampening effects from active air circulation. Most of the recent studies have investigated particle dispersion in a negative-pressure room to reduce the ambient background $[11,16,18]$, but this does not completely exclude ambient particles that potentially can contribute to the measurements. WiLson et al. [15] used zeroing of particle levels between measurements like we did.

The airflows used for HFNC and LFNC were similar to what is typically used in the clinic, and to what have been used in recent studies, while our setup with a mesh of sensors in the test chamber was more extensive than other recent studies [11, 14-16, 18]. Gaeckle et al. [11] and Wilson et al. [15] used funnels to accurately capture all dispersed aerosols, but this approach does not allow for the ability to measure how aerosols spread spatially from the subject. Вем et al. [16] measured at the four cardinal directions from the subject during HFNC, capturing particle dispersion in a circle around the subject at $30 \mathrm{~cm}$ and $1 \mathrm{~m}$ when using HFNC $\left(60 \mathrm{~L} \cdot \mathrm{min}^{-1}\right)$, but to achieve the spatial resolution they measured eight separate times with a handheld instrument. We measured simultaneously at 10 different locations to the sides and in front of the subject for a distance up to $2.85 \mathrm{~m}$ (figure 1). Our results corroborate the results reported by $\mathrm{BEM}$ et al. [16] in that there were no significant differences in particle levels between LFNC and HFNC for the particle range below $5 \mu \mathrm{m}$.

While we found few differences in particle levels versus different distances for LFNC, HFNC and NIV without coughing, we did detect a difference in particle levels as a function of distance for episodes 

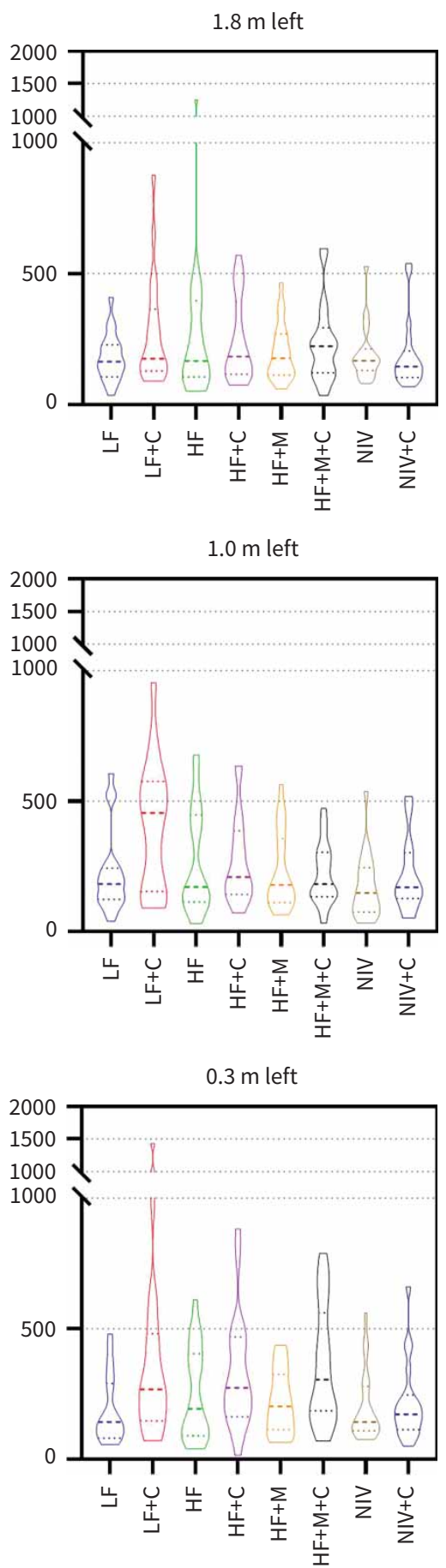
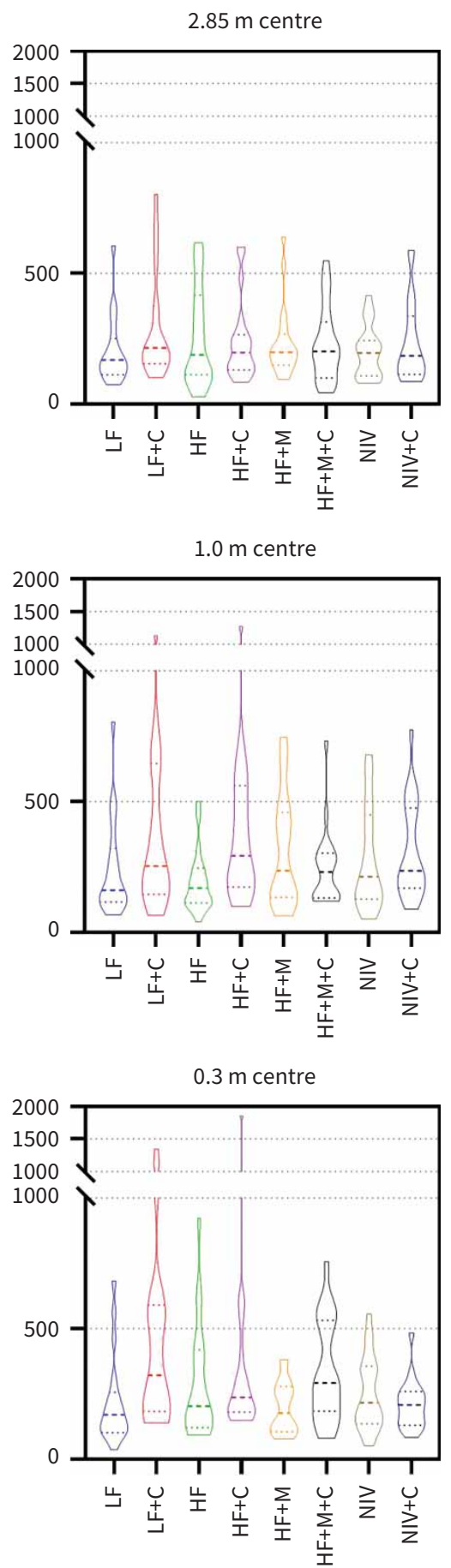
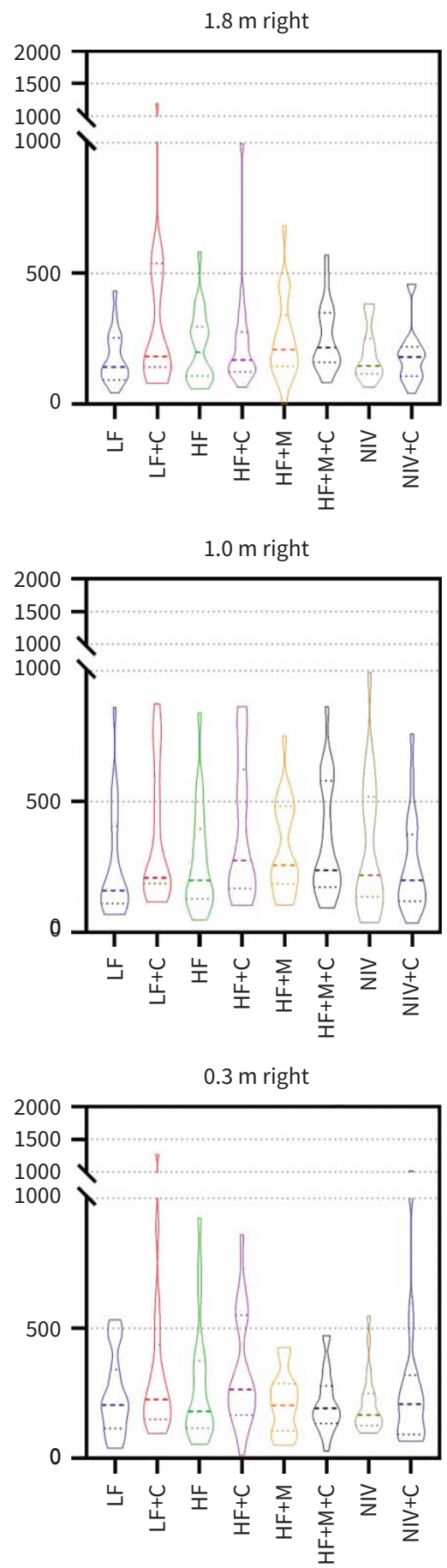

FIGURE 4 Mean particle concentration in particles per litre of air ( $y$-axes) within each episode, for all mesh sensors. The particle concentration number includes particle sizes $(0.3-10 \mu \mathrm{m})$. The placement of the plots within the graph is similar to the sensor position during the experiments, with the distance to each sensor shown in the subfigure headings. The distribution of measurements between participants is presented as violin plots, where the median is indicated with a thin black dashed line, and the quartiles with thinner dashed lines. LF: low-flow; C: cough; HF: high-flow; M: mask; NIV: noninvasive positive-pressure ventilation.

involving coughing. LFNC+cough and HFNC+cough led to relatively large increases in particle concentration close to the subjects (table 3, figure 3), indicating how coughing can lead to a build-up of aerosol particles (10-min period) in an enclosed space with no air circulation, where aerosol concentration drop-off occurred between $100 \mathrm{~cm}$ and $180 \mathrm{~cm}$ (figure 4, table 4). In a hospital setting there will typically be an active room ventilation creating airflows moving in the room. The turbulences formed by these air 
currents will vary with each location. Consequently, making exact predictions about safe distances are difficult. If the clinical setting involves low air-change rates like in a standard patient room, our results indicate that a clinician treating (LFNC or HFNC) a coughing patient may be exposed to significantly lower particle levels at $180 \mathrm{~cm}$ distance compared to a $100-\mathrm{cm}$ distance or closer.

There was a significant reduction in measured particle levels when comparing HFNC+cough or LFNC +cough with NIV+cough, indicating that for a patient with non-productive cough, NIV with double-limb circuit and non-vented mask can be a favourable choice to reduce aerosol dispersal. For patients with productive cough, other modalities may be favourable. GAECKLE et al. [11] reported that NIV might have a dampening effect on aerosol dispersal. WILson et al. [15] reported a lower increase in particles when using single-limb NIV compared to dual-limb NIV (we used dual-limb).

Wearing a surgical mask (HFNC+mask+cough) had a dampening effect on the large cough-spikes of particles in the medium $(>1 \mu \mathrm{m}$ and $\leqslant 5 \mu \mathrm{m})$ and large $(>5 \mu \mathrm{m})$ particle size range (supplementary Figures E1-E3). As a comparison, WiLson et al. [15] reported that using a surgical mask halved the number of particles measured in most instances they tested when using HFNC and NIV on 10 healthy volunteers.

Our primary interest was in the $0.3-5.0 \mu \mathrm{m}$ particle size range (figure $2 \mathrm{e}$ ), which are typically the dehydrated remains from slightly larger primary respiratory droplets. While particles in this size range represent only a fraction of the total volume emitted from the respiratory tract and have lower viral load potential than the larger particles, they are emitted in huge numbers compared to the larger particles [19] and can remain airborne for longer periods of time (hours) under all temperature and relative humidity conditions [20]. At the same time, particles in this range are known as the "breathable/inhalable fraction" and will typically reach and be deposited in the bronchioles and the alveoli of the lower airways [21]. The smallest particle size $(0.3 \mu \mathrm{m})$ in the range we measured (figure 2e) is known as "the most penetrating particle size" due to the difficulty of capturing this particle size with particle filters [22].

\section{Limitations}

This was an explorative study, where the effect sizes and individual variations were unknown prior to the study. Owing to the large individual variation, the study sample size $(\mathrm{n}=20)$ may have limited the power to detect true differences between groups. For future studies we recommend including a larger number of subjects to better account for individual variation. Patients with respiratory disease might have changes in airway parameters, resulting in different aerosol generation characteristics than from healthy adult subjects [23]. Thus, our results might underestimate aerosol dispersal compared to people affected by respiratory disease. Our results in a controlled chamber might not apply for a clinical setting, where room ventilation frequencies and sizes are different.

Provoked coughing by healthy adults may be a poor representation of actual aerosol dispersal by patients that have airway-related diseases. Also, there was large individual variation in coughing intensity. Although the measurements of the low-cost mesh sensors tended to agree with the more precise breathing-zone single sensor, the results based on these sensors are probably less accurate [24]. While we aimed at creating a similar setting for each subject, there were minor variations in sitting positions and the angles between the breathing-zone of the subjects and the sensors that might have influenced the accuracy of the particle measurements. The poor ability for the SPS30 sensor to separate particle sizes in bins [24] was a limitation for our ability to discern how different particle sizes propagate spatially.

\section{Conclusion}

In the study group of 20 healthy individuals, using HFNC and NIV did not lead to increased aerosol dispersal compared to low-flow oxygen treatment, except in rare cases. For a subject with non-productive cough, NIV with double-limb circuit and non-vented mask may be a favourable choice to reduce the risk for aerosol spread.

Acknowledgements: The authors are grateful for the contributions from the participants in the study, the Department of Respiratory Medicine, the Department of Anesthesiology at Oslo University Hospital, the biostatistician Jon Michael Gran, and Gunnar Skogan at the Norwegian Defence Research Establishment.

Author contributions: R. Strand-Amundsen, C. Tronstad, O. Elvebakk, T. Martinsen, M. Dybwad, E. Lingaas and T.I. Tønnessen designed the study, analysed the results, and wrote and edited the article. O. Elvebakk included the healthy volunteers. R. Strand-Amundsen, T.I. Tønnessen, M. Dybwad and E. Lingaas allocated equipment, resources and facilities. C. Tronstad performed the statistical analysis. R. Strand-Amundsen, C. Tronstad, O. Elvebakk and T. Martinsen performed the experiments. All authors read and approved the final manuscript. 
Provenance: Submitted article, peer reviewed.

Availability of data and materials: The datasets used and/or analysed during the current study are available from the corresponding author on request.

Conflict of interest: None declared.

\section{References}

1 Demoule A, Vieillard Baron A, Darmon M, et al. High-flow nasal cannula in critically III patients with severe COVID-19. Am J Respir Crit Care Med 2020; 202: 1039-1042.

2 Mellado-Artigas R, Ferreyro BL, Angriman F, et al. High-flow nasal oxygen in patients with COVID-19-associated acute respiratory failure. Crit Care 2021; 25: 58.

3 Tran K, Cimon K, Severn M, et al. Aerosol generating procedures and risk of transmission of acute respiratory infections to healthcare workers: a systematic review. PLOS ONE 2012; 7: e35797.

4 Jayaweera M, Perera H, Gunawardana B, et al. Transmission of COVID-19 virus by droplets and aerosols: a critical review on the unresolved dichotomy. Environ Res 2020; 188: 109819.

5 Zhang R, Li Y, Zhang AL, et al. Identifying airborne transmission as the dominant route for the spread of COVID-19. Proc Natl Acad Sci USA 2020; 117: 14857-14863.

6 Smith SH, Somsen GA, van Rijn C, et al. Aerosol persistence in relation to possible transmission of SARS-CoV-2. Phys Fluids (1994) 2020; 32: 107108.

7 Iwashyna TJ, Boehman A, Capelcelatro J, et al. Variation in aerosol production across oxygen delivery devices in spontaneously breathing human subjects. medRxiv 2020; preprint [https://doi.org/10.1101/2020.04.15. 20066688].

8 Miller DC, Beamer P, Billheimer D, et al. Aerosol risk with noninvasive respiratory support in patients with COVID-19. J Am Coll Emerg Physicians Open 2020; 1: 521-526.

9 Takazono T, Yamamoto K, Okamoto R, et al. Effects of surgical masks on droplet dispersion under various oxygen delivery modalities. Crit Care 2021; 25: 89.

10 Leung $\mathrm{CCH}$, Joynt GM, Gomersall CD, et al. Comparison of high-flow nasal cannula versus oxygen face mask for environmental bacterial contamination in critically ill pneumonia patients: a randomized controlled crossover trial. J Hosp Infect 2019; 101: 84-87.

11 Gaeckle NT, Lee J, Park Y, et al. Aerosol generation from the respiratory tract with various modes of oxygen delivery. Am J Respir Crit Care Med 2020; 202: 1115-1124.

12 Li J, Fink JB, Ehrmann S. Author's reply on high-flow nasal cannula for COVID-19 patients: low risk of bio-aerosol dispersion. Eur Respir J 2020; 56: 2003136.

13 Haymet A, Bassi GL, Fraser JF. Airborne spread of SARS-CoV-2 while using high-flow nasal cannula oxygen therapy: myth or reality? Intensive Care Med 2020; 46: 2248-2251.

14 Helgeson SA, Lee AS, Lim KG, et al. Particulate generation with different oxygen delivery devices. Respir Med 2021; 181: 106386.

15 Wilson NM, Marks GB, Eckhardt A, et al. The effect of respiratory activity, non-invasive respiratory support and facemasks on aerosol generation and its relevance to COVID-19. Anaesthesia 2021; 76: 1465-1474.

16 Bem RA, van Mourik N, Klein-Blommert R, et al. Risk of aerosol formation during high-flow nasal cannula treatment in critically ill subjects. Respir Care 2021; 66: 891-896.

17 Li J, Fink JB, Elshafei AA, et al. Placing a mask on COVID-19 patients during high-flow nasal cannula therapy reduces aerosol particle dispersion. ERJ Open Res 2021; 7: 00519-2020.

18 Li J, Fink JB, Ehrmann S. High-flow nasal cannula for COVID-19 patients: risk of bio-aerosol dispersion. Eur Respir J 2020; 55: 2000892.

19 Fennelly KP. Particle sizes of infectious aerosols: implications for infection control. Lancet Respir Med 2020; 8 : 914-924.

20 Åkervik E, Fossum H, Dybwad M, et al. Airborne transmission of viral respiratory infections from an aerosol physics perspective. https://www.ffi.no/en/publications-archive/luftbaren-smitte-av-virale-luftveisinfeksjonerfra-et-aerosolfysisk-perspektiv Date last accessed: June 2020.

21 Jackson W. Nebulised Budesonide Therapy in Asthma: A Scientific and Practical Review. UK, Clinical Vision Ltd, 1995.

22 Hinds WC. Aerosol Technology: Properties, Behavior, and Measurement of Airborne Particles, 2nd edn. New York, Wiley-Interscience, 1999.

23 Lindsley WG, Pearce TA, Hudnall JB, et al. Quantity and size distribution of cough-generated aerosol particles produced by influenza patients during and after illness. J Occup Environ Hyg 2012; 9: 443-449.

24 Kuula J, Makela T, Aurela M, et al. Laboratory evaluation of particle-size selectivity of optical low-cost particulate matter sensors. Atmos Meas Tech 2020; 13: 2413-2423. 